\title{
Grey System Theory Based Net Load Forecasting for High Renewable Penetrated Power Systems
}

\author{
Sreenu Sreekumar ${ }^{1} \cdot$ Kailash Chand Sharma $^{2} \cdot$ Rohit Bhakar $^{3}$ (I) \\ Received: 4 December 2019 / Accepted: 6 October 2020 / Published online: 31 October 2020 \\ (C) Springer Nature Singapore Pte Ltd. 2020
}

\begin{abstract}
Net-load is the imbalance between aggregated load and renewable generation. System operations like economic dispatch necessitate accurate forecasting of net-load. There has been significant progress in load and renewable generation forecasting, however, little research focus has been there on Net-Load Forecasting (NLF). Therefore, this paper proposes three Direct Grey index net-load forecasting models (DGM (1, 1), DGM (1, 2) and DGM $(1,3))$ using Grey System Theory (GST). GST based models are suitable for accurate and fast very short-term (five minutes ahead) forecasting due to their momentum transfer behavior. Proposed NLF models are implemented for the Bonneville Power Administration (BPA) balancing area. Forecasts obtained from proposed models are compared with actual net load data and forecasts obtained from reference Artificial Neural Network (ANN) model. Comparison with actual net load shows that proposed models have strong potential for very short-term NLF. At very short time frames, net load shows a very high correlation with previous time steps data. Proposed models utilize such characteristics for forecasting, compared to continuous error reduction procedure in ANN. Continuous error reduction in a very short time frame can lead to under/overestimation of ANN weights and that lowers the forecasting accuracy. Proposed NLF models, especially DGM $(1,3)$ show superior performance over ANN.
\end{abstract}

Keywords Aggregated load forecasting · Grey system theory · Net load forecasting · Power system planning · Solar generation forecasting · Wind generation forecasting

\section{Introduction}

Increasing penetration of uncertain and variable renewable energy such as wind and solar in modern power system enhances uncertain variables in system operational planning. This necessitates complex operational strategies to handle such multivariate uncertainties. Various uncertain variables such as aggregated load, wind and solar power can be combined to form a single uncertain variable, called net load, and can be used to reduce operational

Rohit Bhakar

rbhakar.ee@mnit.ac.in

1 Electrical Electronics Engineering Department, National Institute of Technology Trichy, Tiruchirappalli, India

2 Electrical Engineering Department, Dr. B. R. Ambedkar National Institute of Technology Jalandhar, Jalandhar, India

3 Electrical Engineering Department, Malaviya National Institute of Technology Jaipur, Jaipur, India complexity. Net load is the difference between aggregated system load and renewable generation [21]. Therefore, conventional generators have to be scheduled for net load and power system operational planning like generation flexibility requirement evaluation is planned based on net load [17]. These operations necessitate accurate net load predictions.

Load was the only uncertain operational variable in conventional power systems and operations like economic load dispatch were dependent on load forecasts. This enhanced load forecasting accuracy [1, 25, 28]. Radial basis function based neural network [1], least square support vector machine [28] and hybrid algorithms such as mixed integer programming and genetic algorithm based aggregated tool [25] are widely used for short term load forecasting. It can be observed that most of these models use neural network, support vector regression and hybrid algorithms as base algorithms for forecasting. These algorithms have limitations for very short-term applications in real time due to time consuming learning and hyperparameter optimization procedures. Massive training data requirements and forecasts biased to input data are other major drawbacks of such models [25]. 
Increasing renewable penetration over the last decades has resulted in significant advancement in renewable power forecasting for wind and solar power, due to operational challenges arising from highly uncertain generations $[4,5$, $11,15]$. Hybrid forecasting model based on Brain storm optimization [15], Gaussian mixture based neural network model [5], probabilistic extreme learning machine [11] and probabilistic multi-model ensemble method [4] are widely used for wind speed/power and solar power forecasting. Similar to load forecasting, these models have limitations for very short-term applications in real time due to massive training data requirements, forecasts biased to input data, time consuming learning and hyperparameter optimization procedures. Attempts are being made to reduce operational complexity and associated forecasting errors arising out of considering three different forecasts of load, wind and solar, by consideration of net load forecast and forecasting errors by power markets $[6,10,18,31]$. In such markets, system operations such as economic dispatch $[16,22]$ and generation ramping requirement estimations [8, 29, 30] are planned based on net load forecasts. Even though accurate net load forecasts are vital in modern power system operational planning, it secured little research attention $[6,10,18,27,31]$. Also, most of the NLF approaches focused on micro grids [18, 31], distribution networks [6] and residential loads [27]. System level net load forecasting has secured little attention in existing literature, even though accurate net load forecasts are essential for various operations such as economic dispatch.

Real time economic dispatch time frames have reduced from day ahead to five minutes in several power markets such as Midcontinent Independent System Operator (MISO) and California Independent System Operator (CAISO), to manage the uncertain variability of increasing renewable penetration $[9,13]$. This necessitates fast and accurate five minute ahead net load forecasts. Such net load forecasts can be obtained from NLF models, which require low quantum of forecasting inputs. NLF models are classified as direct and aggregated NLF [18]. Direct NLF uses Net Load Time Series (NLTS) for obtaining forecasts. NLTS can be obtained from load, wind and solar power times series. Individual load, wind and solar power forecasts and their forecasting errors at each time step are used to obtain aggregated net load forecasts. Aggregation of these forecasts and their expected forecasting errors require complex statistical techniques. Such complex algorithms increase the time required to obtain net load forecasts. Direct NLF can be used to obtain faster very short term net load forecasts due to their lower computational complexity.

With this background, this paper proposes three very short term Direct Grey index NLF Models $\operatorname{DGM}(1,1)$, $\operatorname{DGM}(1,2)$ and $\operatorname{DGM}(1,3))$ based on $\operatorname{GST}$ for accurate net load forecasting. Index $(\mathrm{M}, \mathrm{N})$ of $\operatorname{DGM}(\mathrm{M}, \mathrm{N})$ shows the order of differential equation (M) and the number of input variables $(\mathrm{N})$ used in modeling. $\operatorname{DGM}(1,1)$ is a first order uni-variate forecasting model, which uses previous time step net load data for forecasting. DGM(1, 2) and $\operatorname{DGM}(1,3)$ are first order bi-variate and tri-variate forecasting models, respectively. $\operatorname{DGM}(1,2)$ model uses previous year similar day data along with previous time step data and DGM $(1,3)$ model uses two previous year similar days data along with previous time step data. GST based forecasting models can produce ultra fast and accurate very short term net load forecasts compared to complex machine learning algorithms, as these models require low quantum of forecasting inputs due to their momentum transfer behavior [26]. Proposed GST models are implemented for data collected from BPA balancing area. Seasonal analysis is performed to show the forecasting performance of proposed models throughout the year. Forecasts obtained from proposed models are compared with actual net load data and also compared with forecasts obtained from reference ANN model. Comparison with actual net load data shows that proposed models have strong potential to produce accurate very short term net load forecasts. Backpropagation based ANN is used as a reference model due to its superior learning capability [24]. At very short time frames such as five minute steps, net load shows a strong correlation with previous time steps data. Proposed models utilize such characteristics for forecasting compared to continuous error reduction procedure in ANN. Continuous error reduction in a very short time frame can leads to under or over estimation of ANN weights, which lowers the forecast accuracy. Therefore, forecasting performance comparison with ANN shows that proposed very short term direct NLF models, especially DGM (1, 3) has superior performance over ANN in very short time frames.

Remaining part of the paper is arranged as follows. Mathematical modelling of the proposed GST based model is explained in "Proposed Direct NLF Model". "Forecasting Model Performance Evaluation and Comparison" gives model evaluation parameters and "Results and Discussions" gives seasonal performance of proposed models. "Conclusions" concludes that proposed forecasting models are able to produce net load forecasts with low quantum of training data.

\section{Proposed Direct NLF Model}

GST based forecasting models use trend-based forecasting strategy, where trend depends on the set of most recent data. First order differential equation is used to represent the models, which themselves have time varying coefficients. Those coefficients are continuously changing based on the 
availability of new data and those are obtained by solving first order differential equation using least square method. Three GST based direct NLF models; $\operatorname{DGM}(1,1), \operatorname{DGM}(1$, $2)$ and $\operatorname{DGM}(1,3)$ are proposed depending on the number of input variables used for forecasting. $\operatorname{DGM}(1,1), \operatorname{DGM}(1$, 2) and $\operatorname{DGM}(1,3)$ are uni-variate, bi-variate and tri-variate forecasting models, respectively. These models use previous time steps and previous years similar days net load data as forecasting inputs. Historical similar days are used along with previous time steps data to absorb net load behaviour in similar climatic conditions. This can improve forecasting accuracy.

Historical load, wind and solar generation data can be used for historical NLTS estimation. Therefore, proposed very short term direct NLF starts with five minutes interval historical NLTS estimation. Second step obtains very shortterm net load forecasts using three GST based forecasting algorithms (DGM $(1,1)$, DGM $(1,2)$ and DGM $(1,3))$ and third step compares forecasting performance parameters as shown in Fig. 1.

\section{Historical NLTS Estimation}

Net load $\left(N L_{t}\right)$ at each time step is estimated as the difference between aggregated system load $\left(L_{t}\right)$ and sum of aggregated wind $\left(W G_{t}\right) \&$ solar power generation $\left(S G_{t}\right)$ at same time step as follows [12],

$N L_{t}=L_{t}-\left(S G_{t}+W G_{t}\right)$
Historical NLTS data obtained from Eq. 1 is applied to the following GST based Grey index models for obtaining very short term net load forecasts.

\section{Grey Index Models}

Model selection is the primary task for every forecasting problem and it should consider type of data used for forecasting. Different techniques like ACF can be used for forecasting model selection. ACF based model adequacy test is conducted in the proposed work. Very short term forecasting time frame varies from fraction of minutes to several minutes. NLTS shows high auto correlation for such time frames. Grey index models are suitable for highly auto correlated data sets as they use momentum transfer behaviour to obtain forecasts. This enables the use of previous data as forecasting input and also reduces quantum of input data required for forecasting. Therefore, accurate very short time frame net load forecasts can be obtained by Grey index models.

Particular day in any year may have almost similar climate compared to previous years' similar days, as year to year climate change is minimal. Therefore, load, wind and solar power generation data at any day show very strong correlation with previous year's similar day's load, wind and solar power generation data, respectively. So, previous year's similar day's net load data can be used, along with previous time steps net load data, for very short term NLF. Proposed uni-variate $(\operatorname{DGM}(1,1))$ model uses
Fig. 1 Proposed direct NLF model
Step I

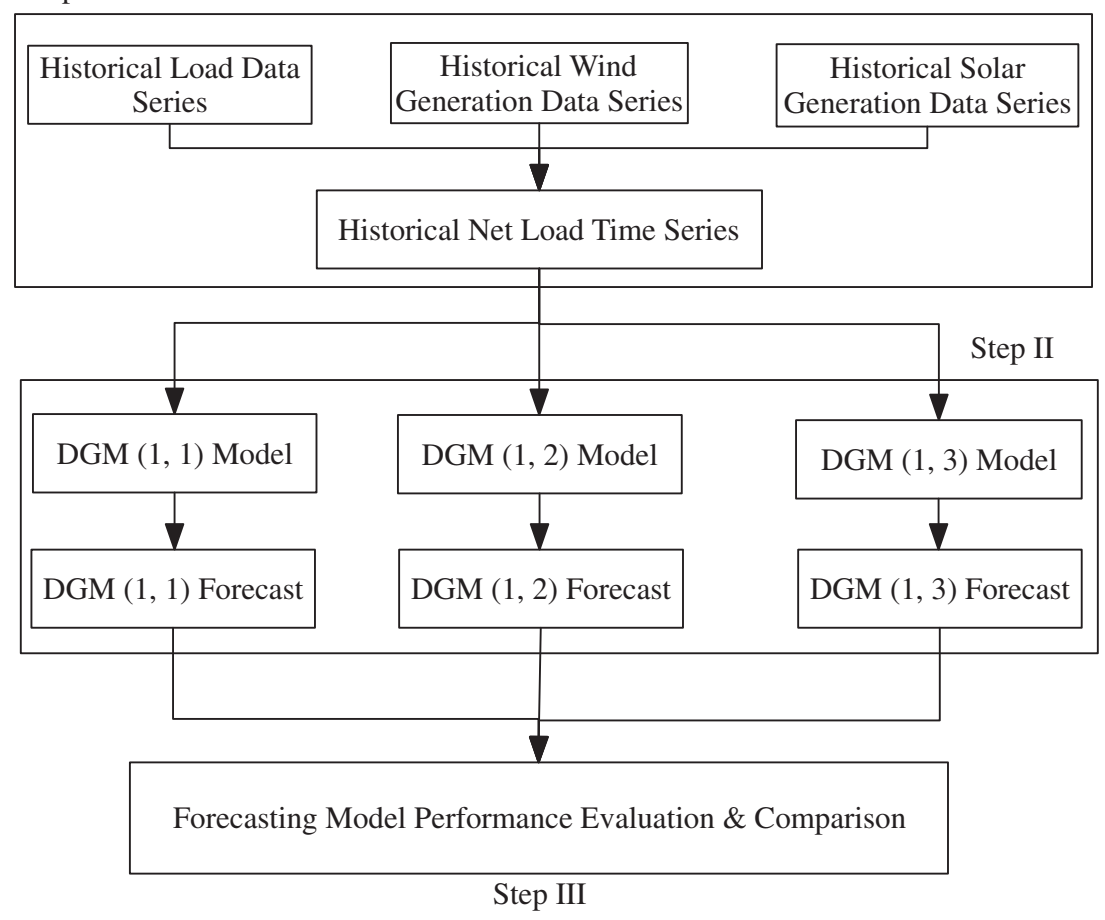


only previous time steps data as forecasting inputs, while bi-variate $(\operatorname{DGM}(1,2))$ and tri-variate $(\operatorname{DGM}(1,3))$ models use previous one year and previous two year data along with previous time steps data, respectively. Grey index models use Accumulated Generated Operation (AGO) as input data pre-processing technique. Model parameters are estimated from AGO sequence. Therefore, model adequacy test is applied on net load AGO sequence. The estimated model creates forecasts as AGO sequence and Inverse Accumulated Generation Operation (IAGO) is required to obtain the actual predicted sequence.

\section{Uni-Variate Grey Model (DGM(1, 1))}

Grey index models are suitable for positive time series data $[7,14,19,20,23,26,33]$. NLTS is positive in most of the power markets as magnitude of base load is higher than sum of maximum possible wind and solar generation [3]. $\operatorname{DGM}(1,1)$ is a first order one variable time series forecasting technique. First order differential equation represents that model has time varying coefficients. Time varying coefficients of model can be obtained by solving first order differential equations.

Proposed model uses AGO as input data processing technique. AGO creates monotonically increasing time series data and such monotonically increasing data shows less randomness as compared to original input data. Proposed model produces forecasts as AGO sequence. IAGO is applied to AGO sequence forecast for obtaining actual forecasts [7, 14, 19, 20, 23, 33].

Consider NLTS data $S^{0}$ that denotes previous $N$ five minute time steps,

$S^{0}(t)=\left(S^{0}(1), S^{0}(2), S^{0}(3), \ldots, S^{0}(N)\right)$

AGO converts NLTS into a monotonically increasing sequence $S^{1}$, which is random as compared to actual data.

$S^{1}(t)=\left(S^{1}(1), S^{1}(2), S^{1}(3), \ldots, S^{1}(N)\right)$

where,

$S^{1}(t)=\sum_{k=1}^{t} S^{0}(k)$

where, $k$ is that time step up to which $S^{1}(t)$ is evaluated. $\operatorname{DGM}(1,1)$ model whitening equation for the previous $N$ time steps net load series can be written as,

$\frac{\mathrm{d}}{\mathrm{d} t} S^{1}(t)+\mu S^{1}(t)=\mu_{1}$

where $\mu$ and $\mu_{1}$ are $\operatorname{DGM}(1,1)$ model parameters. DGM $(1,1)$ first order differential equation least square estimate series can be obtained from previous $N$ time steps net load series $S^{0}$ and generated mean sequence $I^{1}$,

$S^{0}(t)+\mu I^{1}(t)=\mu_{1}$
AGO output of previous N time steps NLTS is used to create generator mean sequence. Such generated mean sequence can be written as,

$I^{1}=\left[I^{1}(1), I^{1}(2), I^{1}(3), \ldots, I^{1}(N)\right]$

where, $I^{1}$ is calculated as the average of subsequent AGO outputs,

$I^{1}(t)=0.5\left(S^{1}(t)+S^{1}(t-1)\right)$

$\operatorname{DGM}(1,1)$ model parameters can be obtained using following matrix operations,

$\left[\begin{array}{ll}\mu & \mu_{1}\end{array}\right]^{T}=\left[\begin{array}{ll}B^{T} & B\end{array}\right]^{-1} \cdot B^{T} . Y$

where,

$Y=\left[S^{1}(2), S^{1}(3), \ldots, S^{1}(N)\right]^{T}$

$B=\left[\begin{array}{cc}-I^{1}(2) & 1 \\ -I^{1}(3) & 1 \\ -I^{1}(4) & 1 \\ \vdots & : \\ -I^{1}(N) & 1\end{array}\right]$

where, $B$ is model matrix. Monotonically increasing forecasted value for next time step $t+1\left(S_{p}^{1}(t+1)\right)$ is given by,

$S_{p}^{1}(t+1)=\left[S^{1}(0)-\frac{\mu_{1}}{\mu}\right] e^{-\mu t}+\frac{\mu_{1}}{\mu}$

Actual net load forecast at $t+1\left(S_{p}^{0}(t+1)\right)$ is obtained from forecasted AGO sequence by applying IAGO [19, 26]. Forecast at $t+1$,

$S_{p}^{0}(t+1)=\left[\left[S^{1}(0)-\frac{\mu_{1}}{\mu}\right] e^{-\mu t}+\frac{\mu_{1}}{\mu}\right]\left(1-e^{\mu}\right)$

In $\operatorname{DGM}(1,1)$ model, $S_{p}^{0}(t+1)$ is obtained using only previous $N$ time steps net load data.

\section{Bi-Variate Grey Model (DGM(1, 2))}

Addition of previous year similar day data along with previous time step's data can help to improve forecasting accuracy, because climatic conditions are almost similar with negligible changes. $\operatorname{DGM}(1,2)$ is a first order bivariate time series forecasting technique, where $N$ previous time steps and previous year similar day net load data are used as forecasting inputs. Previous year similar day's data consists of previous year forecasting time step data and $\mathrm{N}-$ 1 previous five minutes net load data. First order whitening differential equation of proposed $\operatorname{DGM}(1,2)$ model is,

$\frac{\mathrm{d} S_{1}^{0}}{\mathrm{~d} t}+\mu S_{1}^{1}=\mu_{2}-S_{2}^{1}$ 
where $\mu, \mu_{1}$ and $\mu_{2}$ are $\operatorname{DGM}(1,2)$ model parameters. $\operatorname{DGM}(1,2)$ forecasting model parameters can be estimated by following matrix operations:

$\left[\begin{array}{lll}\mu & \mu_{1} & \mu_{2}\end{array}\right]^{T}=\left[\begin{array}{ll}B^{T} & B\end{array}\right]^{-1} \cdot B^{T} . Y$

where,

$Y=\left[S^{1}(2), S^{1}(3), \ldots, S^{1}(N)\right]^{T}$

$B=\left[\begin{array}{cc}-I^{1}(2) & S_{2}^{1}(2) \\ -I^{1}(3) & S_{2}^{1}(3) \\ -I^{1}(4) & S_{2}^{1}(4) \\ : & : \\ -I^{1}(N) & S_{2}^{1}(N)\end{array}\right]$

Monotonically increasing forecasted value for next time step $t+1$ is given by,

$S_{p}^{1}(t+1)=\left(S^{1}(0)-\frac{\mu_{2}}{\mu} S_{2}^{1}(t+1)\right) e^{-\mu t}+\frac{\mu_{2}}{\mu} S_{2}^{1}(t+1)$

Actual net load forecast at $t+1$ is obtained from forecasted AGO sequence by applying IAGO [19, 23]. Forecast at $t+1$,

$S_{p}^{0}(t+1)=S^{1}(t+1)-S^{1}(t)$

In $\operatorname{DGM}(1,2)$ model, $S_{p}^{0}(t+1)$ is obtained using previous time steps and previous year similar day net load data.

\section{Tri-Variate Grey Model (DGM(1, 3))}

Addition of one more previous year's net load data can be used to improve forecasting accuracy, because climatic condition may be different in last year and also may have more similarity with the year before last. $\operatorname{DGM}(1,3)$ is a first order tri-variate time series forecasting technique, which uses previous two years similar day net load data $\left(S_{2}^{0}\right.$ and $\left.S_{3}^{0}\right)$, along with previous time steps net load data $\left(S_{1}^{0}\right)$. Addition of last to last year's similar day data along with previous time steps and previous year similar day data can help to improve forecasting accuracy from $\operatorname{DGM}(1,2)$ model. This will help to dilute the unusual conditions in the just previous year similar day and previous time steps net load data. First order whitening differential equation of $\operatorname{DGM}(1,3)$ model is given as follows:

$\frac{\mathrm{d} S_{1}^{0}}{\mathrm{~d} t}+\mu S_{1}^{1}=\mu_{2}+\mu_{3}-S_{2}^{1}-S_{3}^{1}$

where $\mu, \mu_{1}, \mu_{2}$ and $\mu_{3}$ are $\operatorname{DGM}(1,3)$ model parameters. $\operatorname{DGM}(1,3)$ forecasting model parameters can be estimated by following matrix operations:

$\left[\begin{array}{llll}\mu & \mu_{1} & \mu_{2} & \mu_{3}\end{array}\right]^{T}=\left[\begin{array}{ll}B^{T} & B\end{array}\right]^{-1} \cdot B^{T} . Y$

where,

$Y=\left[S^{1}(2), S^{1}(3), \ldots, S^{1}(N)\right]^{T}$

$$
B=\left[\begin{array}{ccc}
-I^{1}(2) & S_{2}^{1}(2) & S_{3}^{1}(2) \\
-I^{1}(3) & S_{2}^{1}(3) & S_{3}^{1}(3) \\
-I^{1}(4) & S_{2}^{1}(4) & S_{3}^{1}(4) \\
-I^{1}(5) & S_{2}^{1}(5) & S_{3}^{1}(5) \\
\vdots & \vdots & \\
-I^{1}(N) & S_{2}^{1}(N) & S_{3}^{1}(N)
\end{array}\right]
$$

Monotonically increasing forecasted value for next time step $t+1$ is given by,

$$
\begin{array}{r}
S_{p}^{1}(t+1)=S^{1}(0)-\frac{\mu_{2}}{\mu} S_{2}^{1}(t+1) e^{-\mu t}-\frac{\mu_{3}}{\mu} S_{3}^{1}(t+1) e^{-\mu t}+ \\
+\frac{\mu_{2}}{\mu} S_{2}^{1}(t+1)+ \\
\frac{\mu_{3}}{\mu} S_{3}^{1}(t+1)(24)
\end{array}
$$

Actual net load forecast at $t+1$ is obtained from forecasted AGO sequence using IAGO [26]. Forecast at $t+1$,

$S_{p}^{0}(t+1)=S^{1}(t+1)-S^{1}(t)$

Adding more than two year's similar day data may not help to improve accuracy further, because there may be significant changes in load, wind and solar power generation. Significant connected load variation can happen in every year due to the addition of new loads. An average annual load growth rate of 5 to $15 \%$ is present in most of the power industries [3]. Similarly, the installed capacity of wind and solar generation is also rapidly increasing around the world. This also restricts the selection of more than two previous year net load data [3]. Therefore, selecting more than two previous year net load data cannot help to improve the forecasting accuracy.

\section{Forecasting Model Performance Evaluation and Comparison}

Net load forecasts obtained from the above models should be compared with actual net load values for forecasting performance evaluation. Forecasting performance parameters such as Mean Absolute Error (MAE) and Mean Absolute Percentage Error (MAPE) can be used for forecasting performance evaluation [2, 32]. Mean value of absolute errors over an interval can be given as,

$M A E=\frac{\sum \epsilon_{t}}{n}$

Percentage Error (PE) can be estimated as the ratio of absolute error $\epsilon_{t}$ and actual net load $\left(N L_{t}\right)$,

$P E(t)=\frac{\sum \epsilon_{t}}{N L F_{t}} * 100$

MAPE is the arithmetic mean of percentage errors $P E(t)$ over an interval and MAPE can be written as

$M A P E=\frac{\sum P E(t)}{n}$

Performance comparison of proposed Grey index models (DGM $(1,1)$, DGM $(1,2)$ and $\operatorname{DGM}(1,3))$ with an 
Fig. 2 Load, wind \& solar generation and estimated net load data
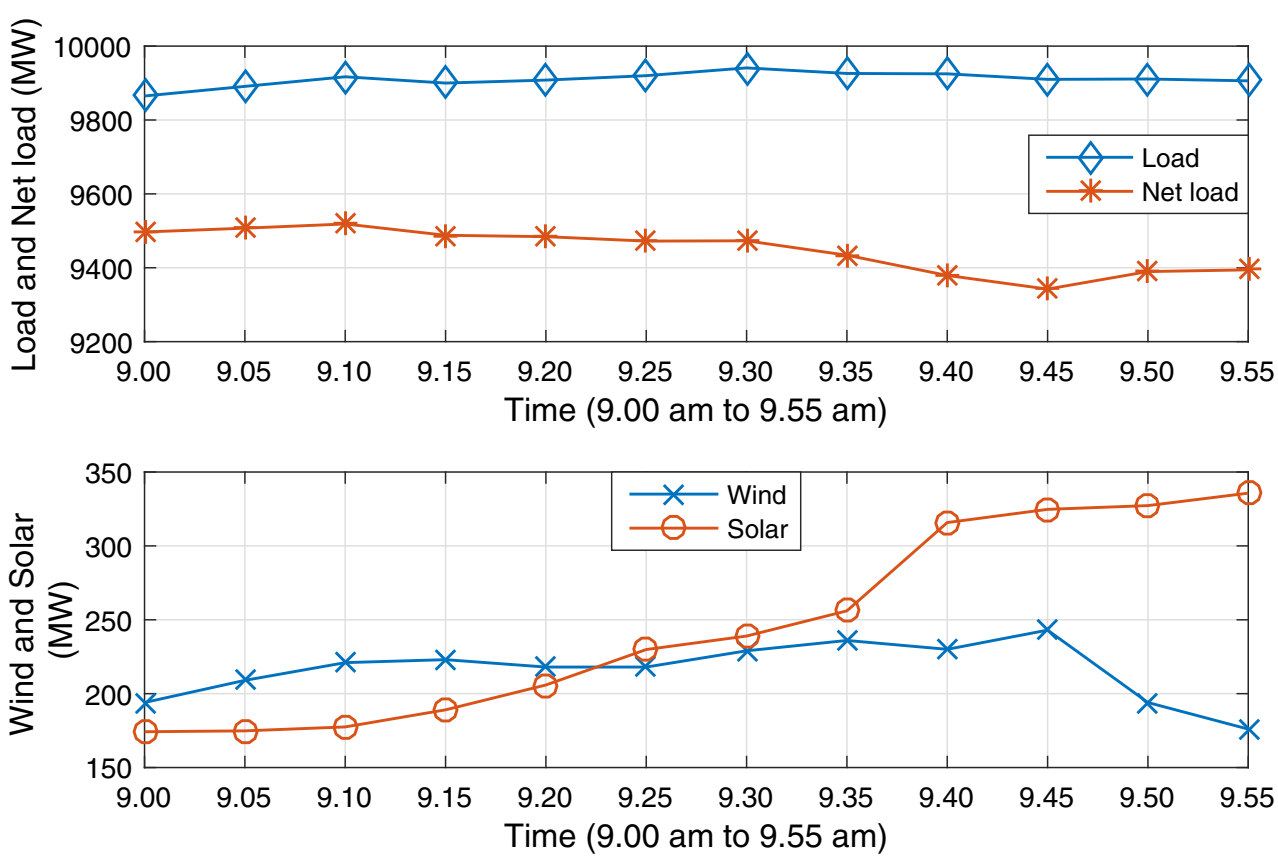

established reference model such as ANN highlights the improvement in forecasting accuracy. ANN is used as a reference model, due to their versatility arising from superior learning capability and forecasting accuracy, as compared to time series models like Auto Regressive Integrated Moving Average Models (ARIMA). Backpropagation based ANN model is implemented for comparison [24].

Optimal performance of proposed model can be ensured by evaluating correlation residues such as ACF. Low correlation and zero mean residues show that no information remains in input net load data, which could be used for improving the forecasting accuracy. Therefore, residual correlation evaluation can be done for proposed models to ensure optimal performance.

\section{Results and Discussions}

Historical NLTS is estimated by Eq. 1 using load, wind and solar power generation data of Bonneville Power Administration (BPA) balancing area [3]. Load and wind power generation data is directly taken from BPA and solar power generation is modelled using solar radiation data. BPA load is composed of aggregated load \& net interchange, and has an average load of 13500 MW. Large number of wind plants are available in BPA balancing area, having an aggregated installed capacity of $4500 \mathrm{MW}$. Aggregated capacity of $1200 \mathrm{MW}$ solar power generation is modelled using solar radiation data, to account globally increasing solar PV generation. Selected system has 5700 MW installed capacity of renewable generation and total renewable generation never reaches to total load, which has an average value of $13500 \mathrm{MW}$. This results in a positive NLTS data for all time steps.

Load, wind generation, solar generation and estimated net load of one morning hour in winter season is shown in Fig. 2. Figure 2 shows that net load is positive for all time steps as renewable generation is too low as compared to system load. Load is slightly increasing for the shown hour as industrial and commercial loads start to build in morning hours. Solar generation also increases due to increasing radiation in morning, while wind generation does not show any trend.

Proposed models obtain point net load forecasts. Figure 3 shows forecasting procedure of proposed point NLF and reference ANN models. It explains training and testing data selection of $\operatorname{DGM}(1,1), \operatorname{DGM}(1,2), \operatorname{DGM}(1,3)$ and reference ANN models. DGM $(1,1)$ model is trained using 2016 data and tested on 2017 data. $\operatorname{DGM}(1,2)$ and both DGM $(1,3)$ as well as ANN are trained using data obtained from 2015, 2016 and 2014, 2015, 2016 years, respectively. Similarly, DGM $(1,2)$ and both $\operatorname{DGM}(1,3)$ as well as ANN are tested using data obtained from 2016, 2017 and 2015, 2016, 2017 years, respectively.

Proposed Grey index models use AGO as data preprocessing technique. AGO transform actual net load time series into monotonically increasing data. Model parameters are evaluated from those monotonically escalating data. Therefore, model adequacy test is conducted on net load AGO data. Model adequacy is evaluated by ACF. ACF plot of net load AGO data is shown in Fig. 4. Figure 4 shows that net load AGO data has moving characteristics and strong auto correlation with previous time steps. So, Grey index models are suitable for net load AGO data forecasting 

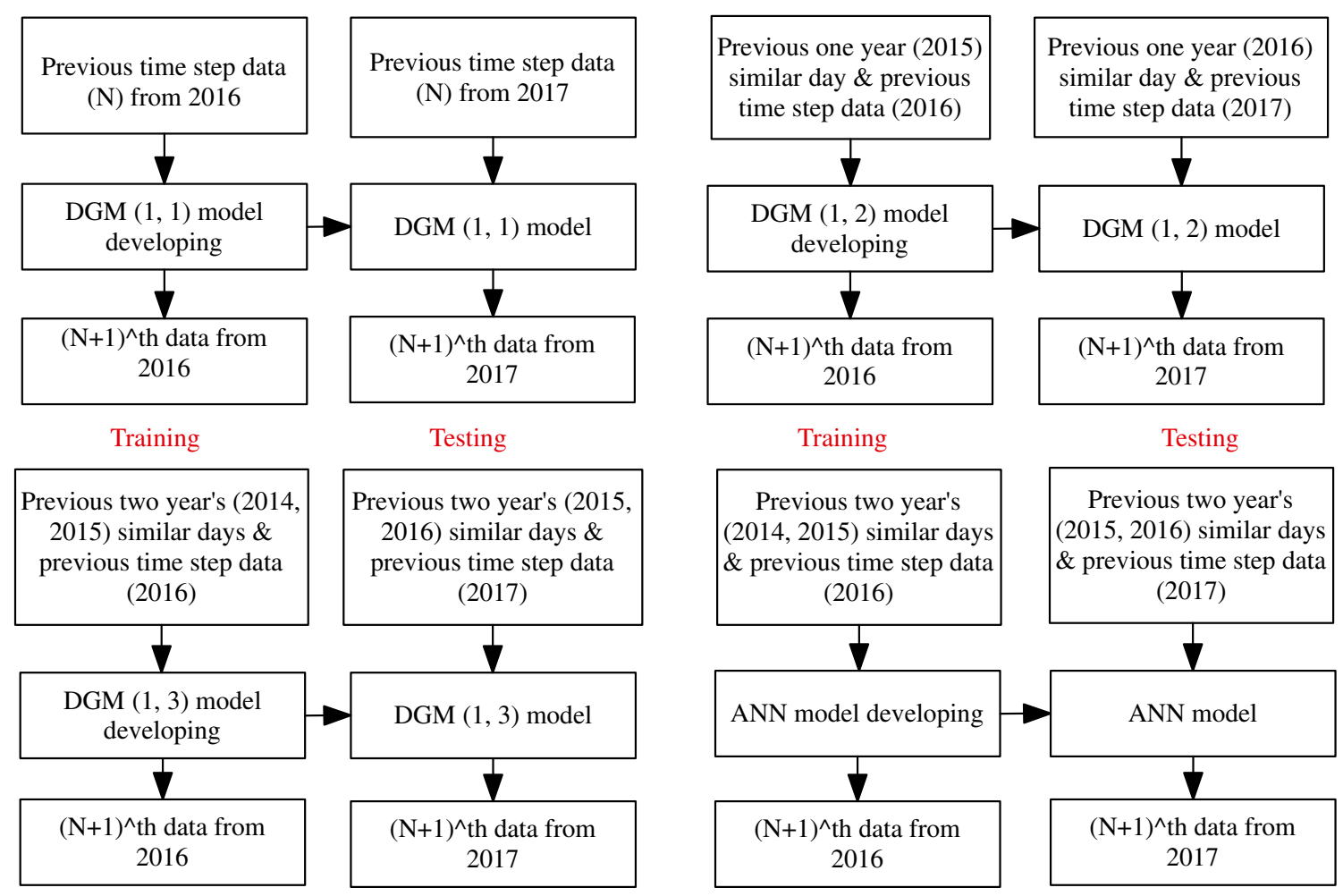

Fig. 3 Forecasting procedure of proposed point NLF and reference ANN models

as those can transfer momentum present in previous time steps data. Proposed model accuracy can also increase by adding previous years similar day net load data as they show almost similar climatic conditions. Similar days are selected based on date, weekday or weekend or holiday information. For obtaining forecasts of weekdays, same date day in the previous year is selected, if it is weekday and nearest date day is selected, if it is weekend. For obtaining forecasts of weekend days, same date day in the previous year is selected, if it is weekend day and nearest date weekend day is selected, if it is weekday. For obtaining

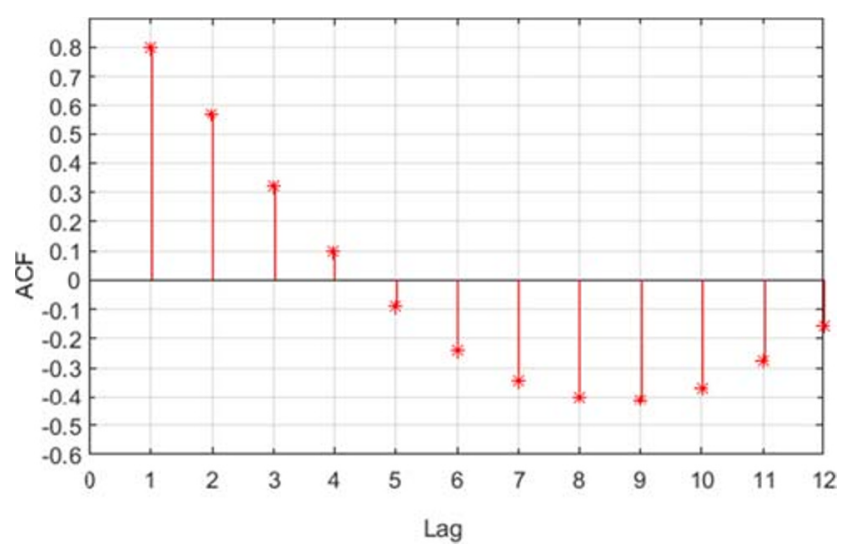

Fig. 4 ACF plot of net load data forecasts of holidays, same holidays are selected from previous years. Addition of previous year's net load data along with previous time steps data as forecasting inputs can be observed in $\operatorname{DGM}(1,2)$ and $\operatorname{DGM}(1,3)$ models. Reference ANN model uses previous two year similar days historical NLTS data and previous time steps data similar to $\operatorname{DGM}(1,3)$ model in all seasons. Proposed Grey index based direct NLF models are implemented for different seasons such as winter, transition and summer.

\section{Winter Season}

Net load forecasts are obtained for morning, noon, evening and night hours of winter season to show the performance throughout the day. Performance plot of one morning hour is shown in Fig. 5. Figure 5 shows that forecasts obtained from Grey index models have same pattern, because previous time step values are common in all Grey index models. Previous years similar day data addition in $\operatorname{DGM}(1,2)$ and $\operatorname{DGM}(1,3)$ models helps to improve the accuracy and it can be observed that forecasts obtained from $\operatorname{DGM}(1,3)$ model are closer to actual values, than any other model. Absolute errors of proposed Grey index models and reference ANN model in complete winter season are shown in Fig. 6.

Error distribution of $\operatorname{DGM}(1,1), \operatorname{DGM}(1,2), \operatorname{DGM}(1$, 3) and ANN models show that forecasting accuracy is improved from $\operatorname{DGM}(1,1)$ to $\operatorname{DGM}(1,3)$. Also, $\operatorname{DGM}(1,3)$ 
Fig. 5 Performance plot of morning hour in winter season

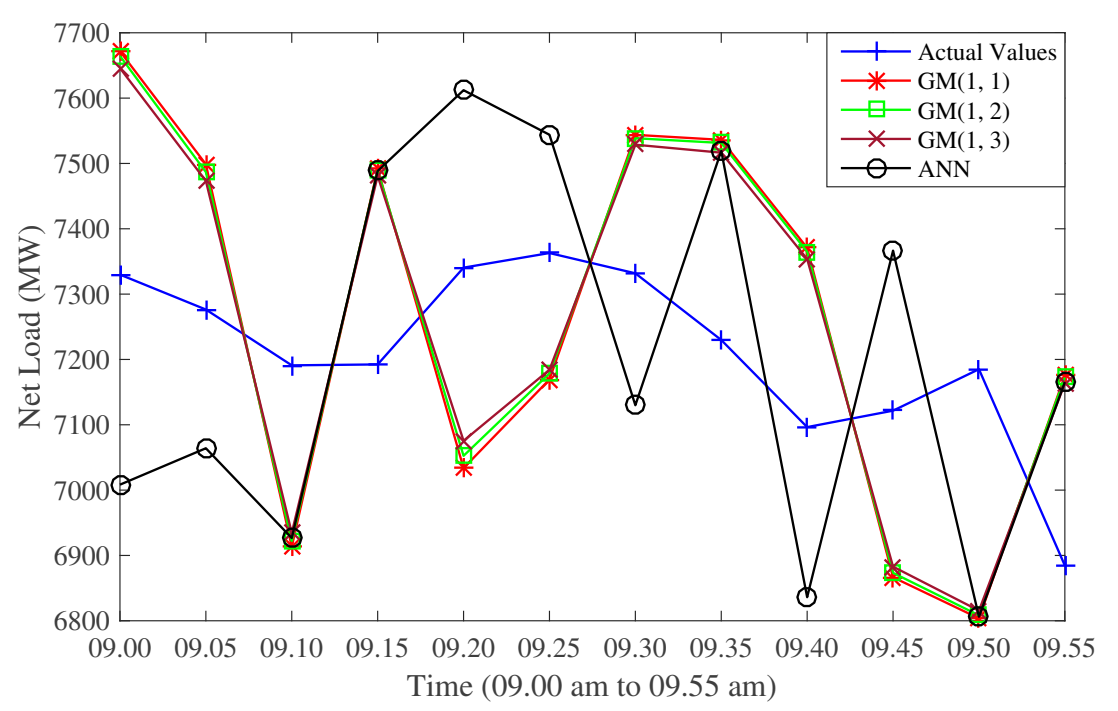

model shows better performance as compared to reference ANN model. Red lines in Fig. 6 show median of absolute errors for different models and it can be observed that $\operatorname{DGM}(1,3)$ model has lowest median. It can also be observed that box's upper border of $\operatorname{DGM}(1,3)$ model is lower than other models. Therefore, $75 \%$ of absolute errors in $\operatorname{DGM}(1,3)$ are distributed closer to zero. However, long upper whisker shows that some extreme absolute errors are present in $\operatorname{DGM}(1,3)$ model. Absolute error distributions can be summarized in terms of forecasting performance parameters. MAE and MAPE of $\operatorname{DGM}(1,1), \operatorname{DGM}(1,2)$, $\operatorname{DGM}(1,3)$ and reference ANN models for morning, noon, evening and night hours in winter season are shown in Table 1.

Table 1 shows that night hours have least MAE values for all models, because net load is the function of only two uncertain variables (load and wind power generation) during night intervals. Night hours also show higher MAPE, because aggregated load is lowest during night hours. Aggregated load in night hours is low due to the absence of industrial and commercial loads, even though heating load is higher. Morning and evening hours show higher MAE and lower MAPE than noon hours, because net load magnitude in noon hours is low due to higher solar power generation and lower heating load. $\operatorname{DGM}(1,1)$ model has an average MAE and MAPE of 311.98 MW and $4.82 \%$ respectively in winter season. $\operatorname{DGM}(1,2)$ model shows $7.91 \%$ and $8.3 \%$ reduction in MAE and MAPE, respectively from $\operatorname{DGM}(1,1)$ model. $\operatorname{DGM}(1,3)$ model shows $21.4 \%$ and $22.88 \%$ reduction in MAE and MAPE, respectively from $\operatorname{DGM}(1,1)$ model. Also, $10.75 \%$ and $11.02 \%$ reduction in MAE and MAPE, respectively is there from
Fig. 6 Absolute error box plot of winter season

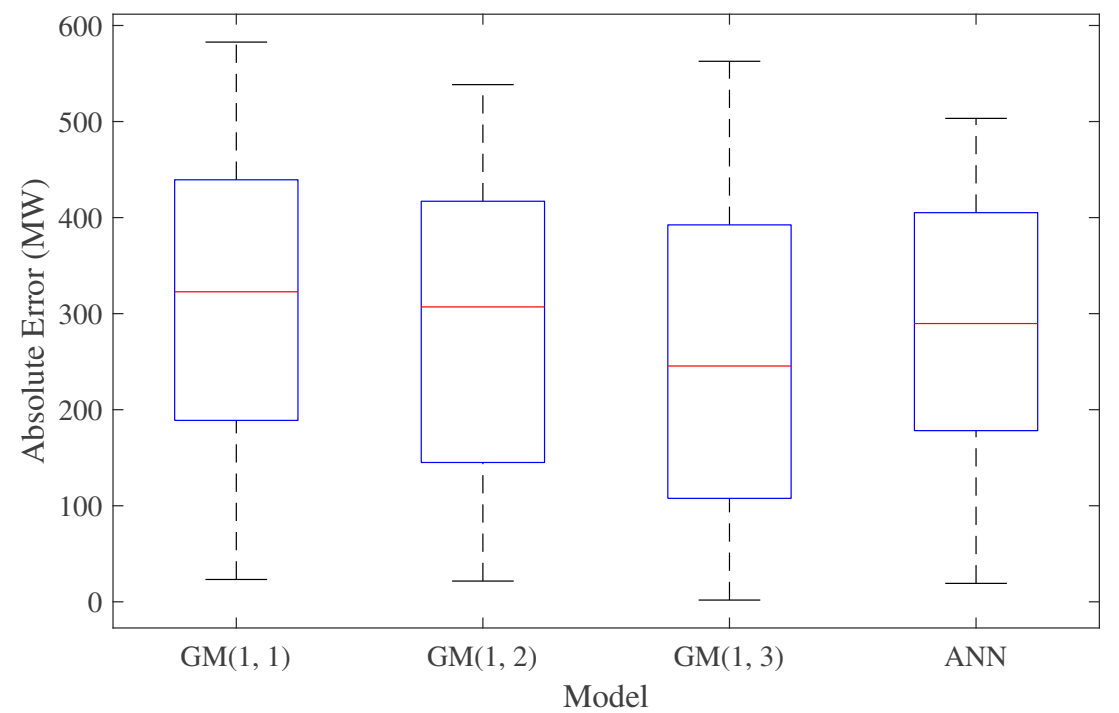


Table 1 MAE (MW) and MAPE (\%) of winter season

\begin{tabular}{|c|c|c|c|c|c|}
\hline Time & Performance Parameter & $\operatorname{DGM}(1,1)$ & $\operatorname{DGM}(1,2)$ & $\operatorname{DGM}(1,3)$ & ANN \\
\hline \multirow[t]{2}{*}{ Morning } & MAE & 335.84 & 327.17 & 260.8 & 318.34 \\
\hline & MAPE & 4.66 & 4.54 & 3.62 & 4.41 \\
\hline \multirow[t]{2}{*}{ Noon } & MAE & 324.37 & 303.24 & 289.9 & 299.14 \\
\hline & MAPE & 4.94 & 4.62 & 4.26 & 4.42 \\
\hline \multirow[t]{2}{*}{ Evening } & MAE & 329.49 & 296.74 & 277.4 & 294.37 \\
\hline & MAPE & 4.68 & 4.22 & 3.95 & 4.19 \\
\hline \multirow[t]{2}{*}{ Night } & MAE & 258.22 & 229.27 & 199.8 & 226.63 \\
\hline & MAPE & 5.01 & 4.45 & 3.86 & 4.4 \\
\hline
\end{tabular}

reference ANN model to $\operatorname{DGM}(1,3)$ model. Therefore, it is clear that proposed $\operatorname{DGM}(1,3)$ model outperforms other models in winter season.

\section{Transition Season}

Morning, noon, evening and night hours of transition season are also selected to show the performance throughout the day. Similar to winter season, performance plot of one morning hour is shown in Fig. 7. Transition season performance plot also follows the pattern of winter season. Net load magnitude of transition season is lower compared to winter season due to higher solar power generation and absence of heating load. Lower net load magnitude results in higher MAPE even for lower absolute errors. Absolute error distribution of various models in transition season is shown in Fig. 8.

Error distribution of $\operatorname{DGM}(1,1), \operatorname{DGM}(1,2), \operatorname{DGM}(1$, 3 ) and ANN models in transition season also shows that forecasting accuracy is improved from $\operatorname{DGM}(1,1)$ model to $\operatorname{DGM}(1,3)$ model. $\operatorname{DGM}(1,3)$ model shows better performance compared to other models, as box's upper border of $\operatorname{DGM}(1,3)$ model error distribution is lower than other models. This shows that $75 \%$ of absolute errors in $\operatorname{DGM}(1,3)$ model are distributed closer to zero. However, long upper whisker shows that some extreme absolute errors are present in $\operatorname{DGM}(1,3)$ model. Figure 8 also shows that ANN model has least median, but more than $50 \%$ of absolute errors are distributed above the median. Error distribution of different models can be summarized in terms of forecasting performance parameters. MAE and MAPE of $\operatorname{DGM}(1,1), \operatorname{DGM}(1,2), \operatorname{DGM}(1,3)$ and reference ANN models for morning, noon, evening and night hours in transition season are shown in Table 2.

Similar to Tables 1 and 2 also shows that night hours have least MAE as night time net load is the function of only two uncertain variables (load and wind power generation). Night hours show higher MAPE, because aggregated load is lowest during night hours due to the absence of industrial and commercial loads. Noon and evening hours show highest MAE and MAPE, respectively in transition season. MAE is highest in noon, because contribution of all three
Fig. 7 Performance plot of morning hour in transition season

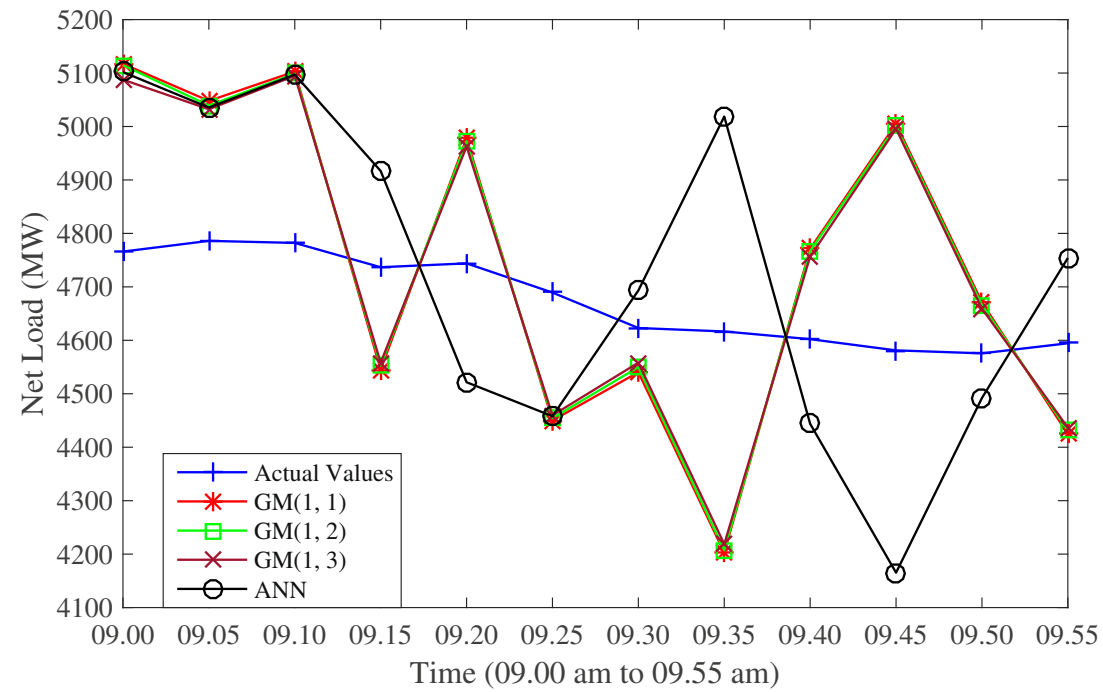


Fig. 8 Absolute error box plot of transition season

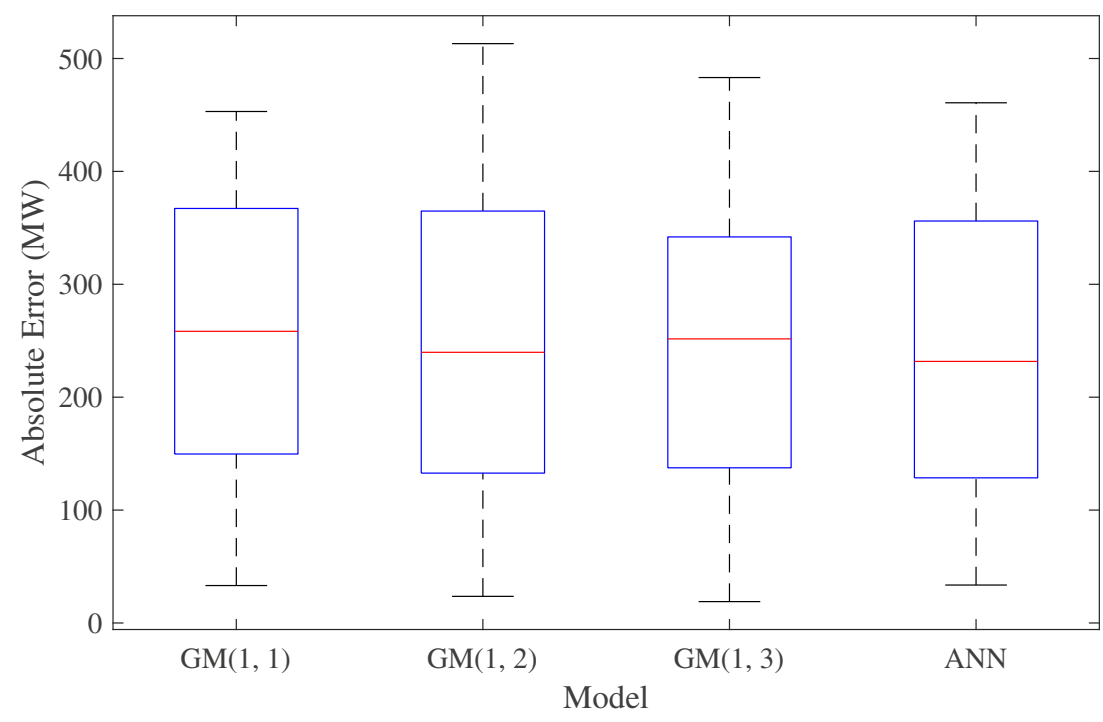

uncertain variables (load, wind and solar power generation) is quite high in net load. MAE magnitudes of morning and evening hours are comparable in transition seasons. However, transition season evening hours show highest MAPE as net load magnitude is low due to high wind power generation. $\operatorname{DGM}(1,1)$ model has an average MAE and MAPE of $256.88 \mathrm{MW}$ and $7.04 \%$ respectively in transition season. $\operatorname{DGM}(1,2)$ model shows $2.55 \%$ and $2.62 \%$ reduction in MAE and MAPE, respectively from $\operatorname{DGM}(1,1)$ model and $\operatorname{DGM}(1,3)$ model shows $5.87 \%$ and $6.02 \%$ reduction in MAE and MAPE, respectively from $\operatorname{DGM}(1,1)$ model. Also, $1.35 \%$ and $1.36 \%$ reduction in MAE and MAPE, respectively is there from reference ANN model to $\operatorname{DGM}(1,3)$ model. Therefore, its clear that proposed $\operatorname{DGM}(1,3)$ model outperforms other models in transition season, similar to winter season.

\section{Summer Season}

Net load forecasts are obtained for morning, noon, evening and night hours of summer season to show the performance throughout the day. Performance plot of one morning hour is shown in Fig. 9. Forecasts obtained by Grey index models show similar pattern, because inputs of all three models contain previous time step net load data. Similar to winter and transition season, use of previous years similar day data as input in $\operatorname{DGM}(1,2)$ and $\operatorname{DGM}(1,3)$ models helped to improve the accuracy. It can be observed that forecasts obtained from $\operatorname{DGM}(1,3)$ model are very close to actual values than other models. Absolute errors of proposed Grey index models and reference ANN model in complete summer season are shown in Fig. 10.

Similar to other seasons, box plot of absolute error shows that forecasting accuracy is improved from $\operatorname{DGM}(1,1)$ model to $\operatorname{DGM}(1,3)$ model. Also, $\operatorname{DGM}(1,3)$ model shows better performance compared to reference ANN model. $\operatorname{DGM}(1,3)$ model has lowest median and also absolute errors are distributed closer to zero than other models. In contrast with other two seasons, $\operatorname{DGM}(1,3)$ model shows shortest whisker in summer season. Therefore, there is no extreme forecasting errors for $\operatorname{DGM}(1,3)$ model in summer season. Absolute error distributions can be summarized in terms of forecasting performance parameters. MAE and MAPE of $\operatorname{DGM}(1,1), \operatorname{DGM}(1,2), \operatorname{DGM}(1,3)$ and reference

Table 2 MAE (MW) and MAPE (\%) of transition season

\begin{tabular}{|c|c|c|c|c|c|}
\hline Time & Performance Parameter & $\operatorname{DGM}(1,1)$ & $\operatorname{DGM}(1,2)$ & $\operatorname{DGM}(1,3)$ & ANN \\
\hline \multirow[t]{2}{*}{ Morning } & MAE & 249.33 & 241.57 & 231.7 & 238.18 \\
\hline & MAPE & 5.31 & 5.15 & 4.94 & 5.07 \\
\hline \multirow[t]{2}{*}{ Noon } & MAE & 315.17 & 311.11 & 302.9 & 303.81 \\
\hline & MAPE & 7.51 & 7.41 & 7.22 & 7.24 \\
\hline \multirow[t]{2}{*}{ Evening } & MAE & 260.22 & 251.87 & 246.3 & 249.17 \\
\hline & MAPE & 7.84 & 7.59 & 7.42 & 7.51 \\
\hline \multirow[t]{2}{*}{ Night } & MAE & 202.81 & 197.34 & 189.6 & 192.44 \\
\hline & MAPE & 7.5 & 7.3 & 7.01 & 7.12 \\
\hline
\end{tabular}


Fig. 9 Performance plot of morning hour in summer season

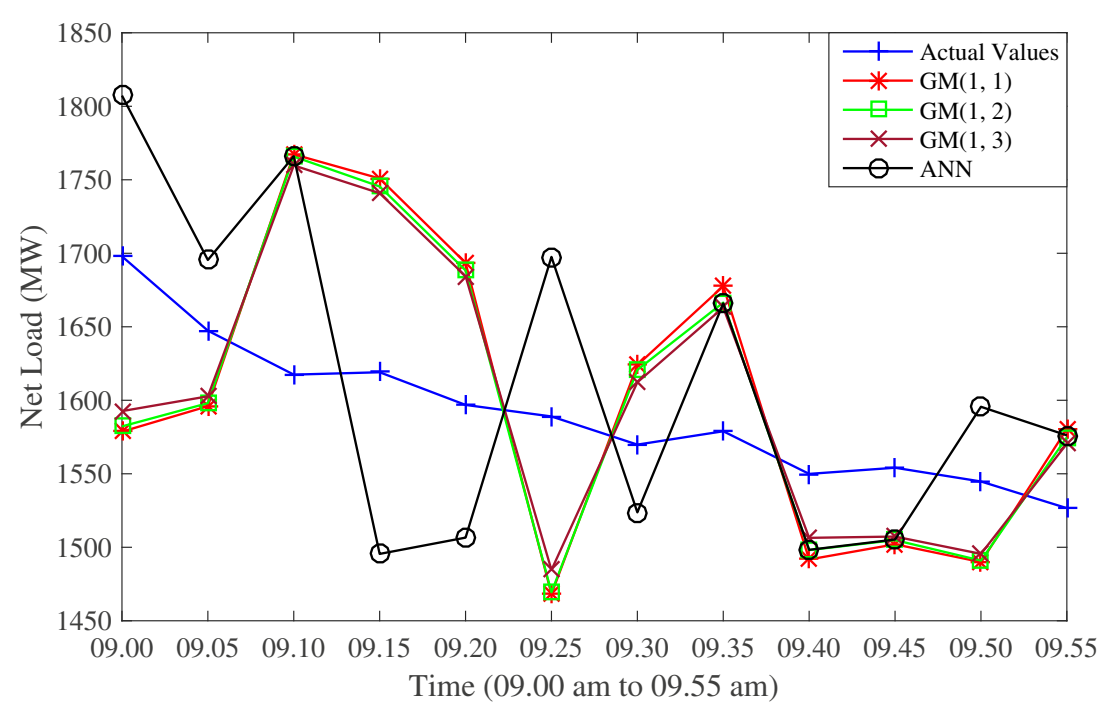

ANN models for morning, noon, evening and night hours in summer seasons are shown in Table 3.

In contrast to winter and transition seasons, summer season night hours show highest MAE and lower MAPE values as night time wind power generation and load are comparatively high. Load is higher due to cooling loads. High wind power generation results in high net load forecasting errors, which leads to higher MAE values. High aggregated load results in higher net load magnitudes and results in lower MAPE values, as actual net load values are present in the denominator of percentage error estimation. This can be observed in Table 3. Noon hours show lowest MAE and comparatively high MAPE due to lower net load magnitudes. $\operatorname{DGM}(1,1)$ model has an average MAE and MAPE of $99.72 \mathrm{MW}$ and $4.77 \%$ respectively in summer season. $\operatorname{DGM}(1,2)$ model shows $12.17 \%$ and
$13.03 \%$ reduction in MAE and MAPE, respectively from $\operatorname{DGM}(1,1)$ model. $\operatorname{DGM}(1,3)$ model shows $19.35 \%$ and 20.15\% reduction in MAE and MAPE, respectively from $\operatorname{DGM}(1,1)$ model. It can also be observed that summer season performance of reference ANN model is almost similar to $\operatorname{DGM}(1,3)$ model. However, there is small reduction of $0.93 \%$ and $0.75 \%$ in MAE and MAPE, respectively from reference $\operatorname{ANN}$ model to $\operatorname{DGM}(1,3)$ model. Therefore, its clear that proposed $\operatorname{DGM}(1,3)$ model outperforms other models in summer season, similar to winter and transition seasons. However, net load forecast trajectory shows slight variation from net load trajectory (minute dynamic variations), because proposed models use momentum of last twelve data points to produce next time step forecast. Forecast may vary if next time step data shows extreme trend variation as compared to recent data.
Fig. 10 Absolute error box plot of summer season

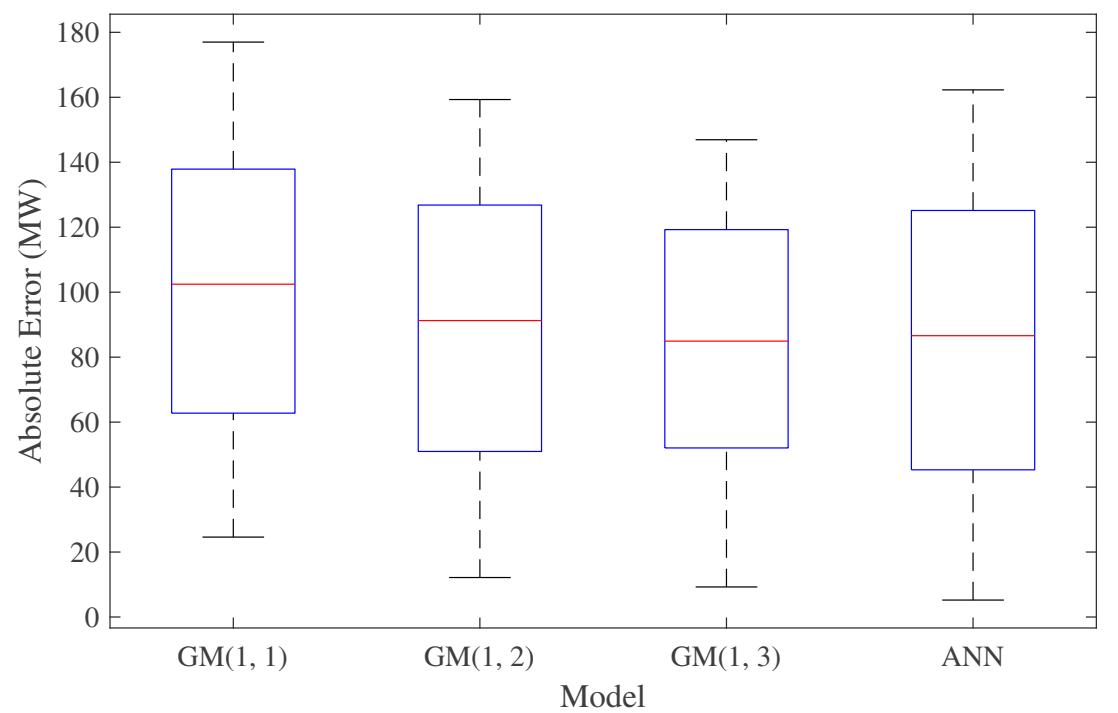


Table 3 MAE (MW) and MAPE (\%) of summer season

\begin{tabular}{llllrr}
\hline Time & Performance Parameter & DGM $(1,1)$ & DGM $(1,2)$ & DGM (1,3) \\
\hline Morn. & MAE & 98.34 & 83.27 & 76.3 & 77.81 \\
& MAPE & 6.13 & 5.19 & 4.76 & 4.85 \\
Noon & MAE & 91.81 & 82.03 & 79.9 & 79.96 \\
& MAPE & 5.44 & 4.86 & 4.74 & 4.74 \\
Even. & MAE & 102.64 & 92.17 & 85.9 & 3.57 \\
& MAPE & 4.26 & 3.83 & 92.1 & 3.59 \\
Night & MAE & 106.11 & 98.14 & 2.82 \\
& MAPE & 3.25 & 3.01 & 93.14 \\
\hline
\end{tabular}

\section{Annual Performance}

Annual performance is evaluated by compiling seasonal analysis. Forecasts obtained in winter, transition and summer seasons are used for annual analysis. Absolute error distribution of $\operatorname{DGM}(1,1), \operatorname{DGM}(1,2), \operatorname{DGM}(1,3)$ and ANN are shown in Fig. 11. Absolute errors successively reduce from $\operatorname{DGM}(1,1)$ model to $\operatorname{DGM}(1,3)$ model. $\operatorname{DGM}(1,2)$ model has lower median compared to ANN model, but absolute errors are quite higher. $\operatorname{DGM}(1,3)$ model has least median compared to other models and also more than $75 \%$ of absolute errors are distributed closer to zero. However, long upper whisker of $\operatorname{DGM}(1,3)$ model shows that some extreme absolute errors are present in $\operatorname{DGM}(1,3)$ model.

Error distribution of different models can be summarized in terms of forecasting performance parameters. MAE and

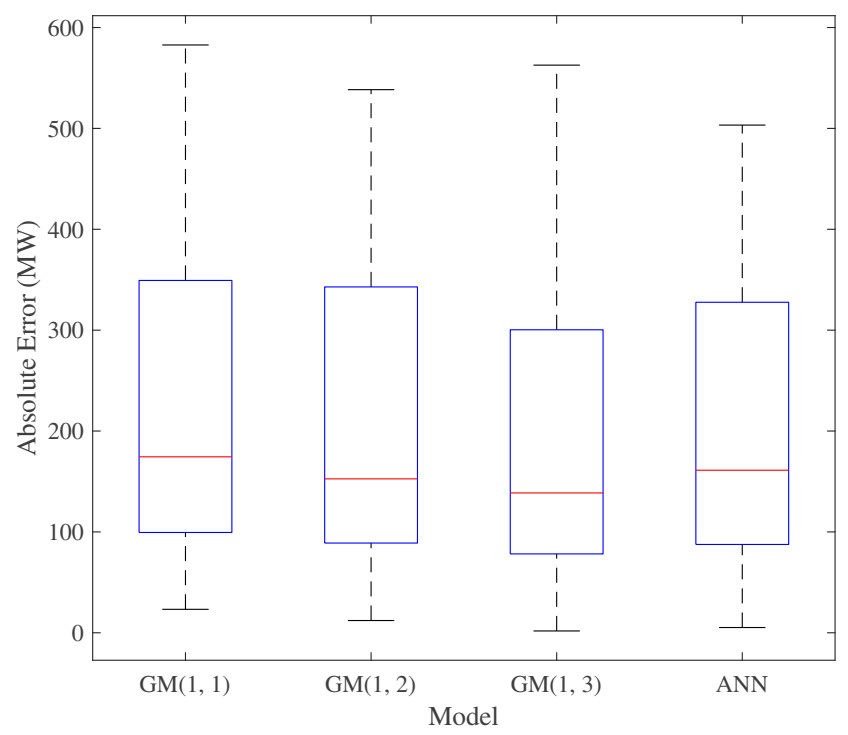

Fig. 11 Annual absolute error box plot
MAPE of $\operatorname{DGM}(1,1), \operatorname{DGM}(1,2), \operatorname{DGM}(1,3)$ and reference ANN models of complete year are shown in Table 4 . $\operatorname{DGM}(1,1)$ model has an average annual MAE and MAPE of $222.86 \mathrm{MW}$ and $5.54 \%$. There is $6.38 \%$ and $6.94 \%$ reduction in MAE and MAPE respectively, from $\operatorname{DGM}(1,1)$ to $\operatorname{DGM}(1,2)$ model. $\operatorname{DGM}(1,3)$ model shows $14.65 \%$ and $14.46 \%$ reduction in MAE and MAPE respectively, from $\operatorname{DGM}(1,1)$ to $\operatorname{DGM}(1,3)$ model. Also, $\operatorname{DGM}(1,3)$ model shows $5.48 \%$ and $3.92 \%$ reduction in MAE and MAPE from reference ANN model. Therefore, it is clear that annually $\operatorname{DGM}(1,3)$ model outperforms other models.

Proposed DGM $(1,3)$ model has an annual accuracy of above $95 \%$ as shown in Table 4 and reflects the capability to produce accurate forecasts. However, proposed models' forecasts show slight deviations from actual net load trajectory (Figs. 7, 8 and 9). Reference ANN model forecasts do not exactly follow the actual net load/ those obtained from the proposed models. Model parameter/weight estimation in proposed Grey index models and reference ANN model is fundamentally different and produce different trend among these. Reference ANN model use backpropagation-based weight adjustment to minimize error.

Proposed models use momentum of last twelve data points to produce next time step forecast and a rolling approach by regularly data updating. Forecast may vary if next time step data shows extreme trend variation as compared to recent data. Proposed models use momentum

Table 4 Annual performance parameters

\begin{tabular}{lll}
\hline Model & MAE $(M W)$ & MAPE $(\%)$ \\
\hline $\operatorname{DGM}(1,1)$ & 222.86 & 5.54 \\
$\operatorname{DGM}(1,2)$ & 209.49 & 5.18 \\
$\operatorname{DGM}(1,3)$ & 194.38 & 4.84 \\
ANN & 204.95 & 5.03 \\
\hline
\end{tabular}


of last 12 data points to produce next time step forecast and a rolling approach by regularly data updating. Forecast may vary if next time step data shows extreme trend variation as compared to recent data. Same can be observed from Figs. 5, 6 and 7. Proposed model forecasts follow increasing and decreasing trend of actual data; however, momentum transfer is leads to overestimated point forecasts. Addition of previous years' similar day data in $\operatorname{DGM}(1,2)$ and DGM $(1,3)$ helps to reduce overestimation and can be observed as accuracy improvement. Also, overestimation tendency is less as compared to reference ANN model.

Proposed models can produce accurate forecasts even with renewable generation changes, as historical renewable generation variations are included in net load time series and model parameters are estimated using such data. However, high uncertainty in renewable generation may enhance net load forecasting error. Positively correlated uncertain changes among load and renewable generation, and negatively correlated wind and solar generation reduces the effect of uncertainty. Negatively correlated uncertain changes among load and renewable generation, and positively correlated wind and solar generation increases the effect of uncertainty.

Residual correlation is estimated for best model $(\operatorname{DGM}(1,3))$ using ACF. ACF of residues of $\operatorname{DGM}(1,3)$ model are shown in Fig. 12. ACF plots show that residues have low correlation; apart from one outlier, all residual correlations are with in 0.2. Also, mean value of residuals is closer to zero and ACF plot does not show any kind of correlation pattern. Therefore, no information remains in input net load data to improve forecasting model performance.

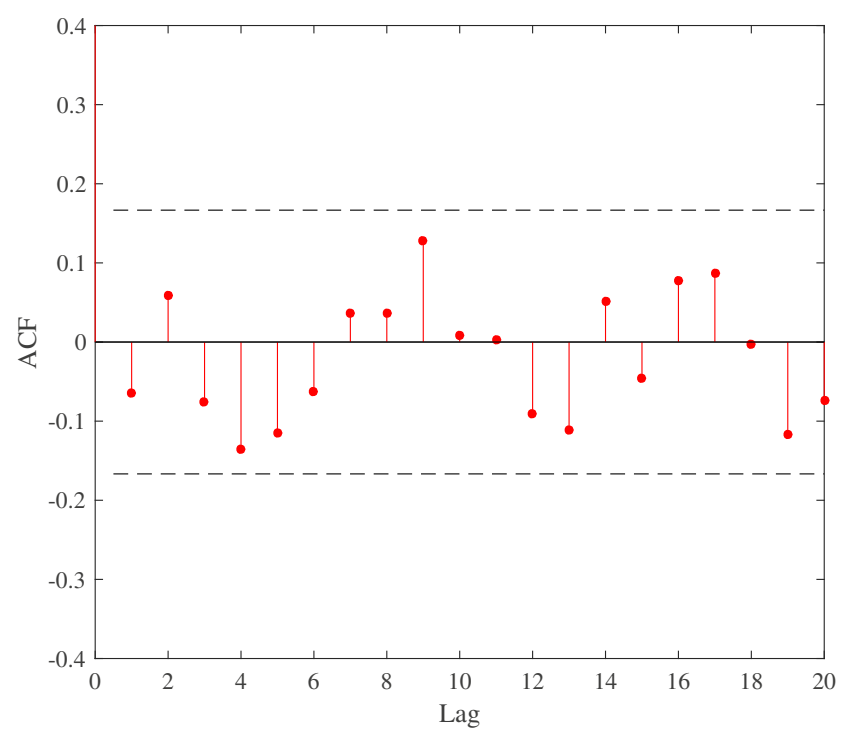

Fig. $12 \mathrm{ACF}$ of $\operatorname{DGM}(1,3)$ model

\section{Conclusions}

High renewable penetrated power systems necessitate accurate net load forecasts for optimal system operations such as generator scheduling and generation ramping requirement estimation. Also, increasing renewable penetration in power systems continuously forces to reduce dispatching times frames from day ahead to fives minutes in markets like MISO and CAISO. This necessitates fast and accurate net load forecasts in such very short time frames. Such fast, accurate and very short time frame net load forecasts can be obtained by direct NLF. Therefore, this paper proposes three very short time frame direct NLF models based on GST. Forecasting performance analysis show that proposed models are suitable to obtain accurate net load forecasts in all seasons. Addition of previous years similar day data along with previous time step data as forecasting inputs helped to improve forecasting accuracy from $\operatorname{DGM}(1,1)$ to $\operatorname{DGM}(1$, 3) model. Proposed $\operatorname{DGM}(1,3)$ model outperforms proposed $\operatorname{DGM}(1,1), \operatorname{DGM}(1,2)$ and reference $\operatorname{ANN}$ model through out the year. Therefore, accurate net-load forecasts obtained from proposed DGM $(1,3)$ model can be used for different power system operations such as generation scheduling. Seasonal analysis shows that summer season shows least error compared to other seasons due to the less uncertain weather.

Direct net load forecasting accuracy can be further improved by avoiding input data outliers using digital signal processing-based data filtering techniques. Also, minute dynamic variations in net load can be addressed using advanced machine learning/deep learning algorithms. Accurate net load forecasts are beneficial for micro/community grid and low voltage network operations, and current work can be extended for such systems. These can be done as future work.

Acknowledgments This work is supported by the DST grant for UKICERI project, DST/RCUK/JVCCE /2015/02.

\section{References}

1. Abedinia O, Amjady N (2017) Short-term load forecast of electrical power system by radial basis function neural network and new stochastic search algorithm. Int Trans Electr Energ Syst 26(7):1511-1525

2. Ali M, Son DH, Kang SH, Nam SR (2017) An accurate ct saturation classification using a deep learning approach based on unsupervised feature extraction and supervised fine-tuning strategy. Energies 10(11):18-30

3. Bonneville Power Administration (2017) Balancing area wind generation and load data. https://transmission.bpa.gov/Business/ Operations/Wind/default.aspx 
4. Bracale A, Carpinelli G, De Falco P (2017) A probabilistic competitive ensemble method for short-term photovoltaic power forecasting. IEEE Trans Sustain Energ 8(2):551-560

5. Chang G, Lu H, Wang P, Chang Y, Lee Y (2017) Gaussian mixture model-based neural network for short-term wind power forecast. Int Trans Electr Energ Syst 27(6):23-30

6. Chu Y, Carlos Coimbra HP (2016) Granular operational net load forecasting. https://www.calsolarresearch.org/images/stories/ documents/Sol4_funded_proj_docs/UCSD4_Kleissl/2_CSI-RDD_ Sol4_UCSD_Task3-GranularOpsNetLoadForeCast-Rpt_2016-04

7. El-Fouly THM, El-Saadany EF, Salama MMA (2006) Grey predictor for wind energy conversion systems output power prediction. IEEE Trans Power Syst 21(3):1450-1452

8. Ela E, O'Malley M (2016) Scheduling and pricing for expected ramp capability in real-time power markets. IEEE Trans Power Syst 31(3):1681-1691

9. FERC (2014) Staff analysis of shortage pricing in RTO and ISO markets. https://www.ferc.gov/legal/staffreports/2014/ AD14-14-pricing-rto-iso-markets.pdf

10. Fonte PM, Monteiro C (2016) Net load forecasting in presence of renewable power curtailment. In: Proceedings international conference on the european energy market, $\mathrm{pp} 1-5$

11. Golestaneh F, Pinson P, Gooi HB (2016) Very short-term non-parametric probabilistic forecasting of renewable energy generation with application to solar energy. IEEE Trans Power Syst 31(5):3850-3863

12. Huang B, Krishnan V, Hodge BM (2018) Analyzing the impacts of variable renewable resources on california net load ramp events. In: Proceedings IEEE power and energy society general meeting, pp 1-5

13. Huber M, Dimkova D, Hamacher T (2014) Integration of wind and solar power in europe: assessment of flexibility requirements. Energy 69:236-246

14. Ji PR, Huang WS, HU XY (2001) A study on the properties of grey forecasting model. Syst Eng Theory Prac 9:1-12

15. Jiang P, Li P (2016) Research and application of a new hybrid wind speed forecasting model on bso algorithm. J Energ Eng 143(1):04016019

16. Jiang $\mathrm{P}$, Huo $\mathrm{Y}, \mathrm{Wu} X$ (2016) A dual timescale active power coordinated scheduling framework for wind integrated power system in the presence of storage and wind forecast uncertainties. Int Trans Electr Energ Syst 26(11):2322-2336

17. Jin S, Botterud A, Ryan SM (2014) Temporal versus stochastic granularity in thermal generation capacity planning with wind power. IEEE Trans Power Syst 29(5):2033-2041

18. Kaur A, Nonnenmacher L, Coimbra CF (2016) Net load forecasting for high renewable energy penetration grids. Energy 114:1073-1084

19. Kayacan E, Ulutas B, Kaynak O (2010) Grey system theory-based models in time series prediction. Expert Syst Appl 37(2):17841789
20. Lee YS, Tong LI (2011) Forecasting energy consumption using a grey model improved by incorporating genetic programming. Energy Convers Manag 52(1):147-152

21. Makarov YV, Etingov PV, Ma J, Huang Z, Subbarao K (2011) Incorporating uncertainty of wind power generation forecast into power system operation, dispatch, and unit commitment procedures. IEEE Trans Sustain Energ 2(4):433-442

22. Nosair H, Bouffard F (2017) Economic dispatch under uncertainty: the probabilistic envelopes approach. IEEE Trans Power Syst 32(3): 1701-1710

23. Ozcan T (2017) Application of seasonal ad multi-variable grey prediction models for short term load forecasting. Alphanumer J pp 329-338

24. Rajihy Y, Nermend K, Alsakaa A (2017) Back-propagation artificial neural networks in stock market forecasting: an application to the warsaw stock exchange wig20. Int J Finance 15:88-99

25. Ruiz N, Claessens B, Jimeno J, López J, Six D (2015) Residential load forecasting under a demand response program based on economic incentives. Int Trans Electr Energ Syst 25(8): 1436-1451

26. Sreenu S, Jatin V, Sujil A, Rajesh K (2016) An approach towards real time short term load forecasting using grey index models for smart grid framework. WSEAS Trans Power Syst 11:149-155

27. Sun M, Zhang T, Wang Y, Strbac G, Kang C (2019) Using bayesian deep learning to capture uncertainty for residential net load forecasting. IEEE Trans Power Syst, https://doi.org/10.1109/TPWRS.2019.2924294

28. Sun W, Liang Y (2014) Least-squares support vector machine based on improved imperialist competitive algorithm in a shortterm load forecasting model. J Energ Eng 141(4):04014037

29. Wang C, Luh PBS, Navid N (2017) Ramp requirement design for reliable and efficient integration of renewable energy. IEEE Trans Power Syst 32(1):562-571

30. Wang Q, Hodge BM (2017) Enhancing power system operational flexibility with flexible ramping products: a review. IEEE Trans Indust Inform 13(4):1652-1664

31. Wang Y, Zhang N, Chen Q, Kirschen DS, Li P, Xia Q (2018) Datadriven probabilistic net load forecasting with high penetration of behind-the-meter pv. IEEE Trans Power Syst 33(3):32553264

32. Wang ZX, Hao P (2016) An improved grey multivariable model for predicting industrial energy consumption in china. Appl Math Model 40(11):5745-5758

33. Yao AW, Chi SC (2004) Analysis and design of a taguchigrey based electricity demand predictor for energy management systems. Energy Convers Manag 45(7):1205-1217

Publisher's Note Springer Nature remains neutral with regard to jurisdictional claims in published maps and institutional affiliations. 